\title{
Avaliação do Uso de Curto-Circuito Controlado em Soldagens de Passes de Enchimento por MIG/MAG Duplo Arame
}

\author{
Dumitru Caimacan ${ }^{1}$, Andrii Mishchenko ${ }^{1}$, Américo Scotti ${ }^{1,2}$ \\ 1 Programa de Pós-Graduação em Engenharia Mecânica, Universidade Federal de Uberlândia - UFU, Uberlândia, MG, Brasil. \\ 2 Grupo Centro para Pesquisa e Desenvolvimento de Processos de Soldagem - LAPROSOLDA, Faculdade de Engenharia \\ Mecânica, Universidade Federal de Uberlândia - UFU, Uberlândia, MG, Brasil.
}

Recebido: 18 Ago., 2014

Aceito: 19 Fev., 2015

E-mails:

dcaimacan@yahoo.com (DC), asmwelder@gmail.com (AM), ascotti@ mecanica.ufu.br (AS)
Resumo: Neste trabalho foi realizada uma avaliação técnico-econômica do processo de soldagem MIG/MAG Duplo Arame para soldagens de passes de enchimento na posição plana, empregando o modo operacional Curto-Circuito Controlado (CCC) no arame-seguidor, como forma de melhorar o controle da poça de fusão e reduzir respingos. A avaliação foi feita através de um estudo comparativo entre a técnica MIG/MAG Duplo Arame convencional (com uso da corrente pulsada em ambos os arames) e uma combinação de Pulsado no arame-líder e CCC no arame-seguidor. Foram mantidos constantes os valores das correntes médias para cada arame, o volume de material depositado por unidade de comprimento de solda, o tipo e dimensão do chanfro e o arame, a composição do gás de proteção e a DBCP. Avaliou-se o acabamento superficial e a geometria dos cordões de soldas, a taxa e a eficiência de deposição e as velocidades limites de soldagem resultantes do uso de cada técnica. Os resultados mostram que o uso do modo CCC no arco seguidor aumenta levemente a capacidade de produção do processo, faz gerar menos respingos, melhora o acabamento do cordão, produz ZF e ZAC menores e ainda mantem similar envelope operacional (quanto à faixa de velocidades de soldagens úteis), o que são fatores positivos. Por outro lado, como desvantagem, o uso de cCC levou a cordões mais convexos e com uma penetração menor.

Palavras-chave: GMAW Tandem; Controle de corrente; Automação.

\section{Assessment of Controlled Short-Circuiting Application in Filling Passes with MIG/MAG Double Wire Process}

\begin{abstract}
In this paper, a techno-economical evaluation of the Double Wire MIG/MAG welding process for filling passes on the flat position was performed, applying Controlled Short-Circuiting (CSC) mode in the trailing wire. The aim was to improve the welding pool control and reduce spatter generation. The evaluation was carried out through a comparative study between the conventional Double Wire MIG/MAG welding technique (using pulsed current in the both wires) and a combination of a pulsed current in the leading wire and CSC in the trailing wire. The values of the average current at each wire, the volume of deposited material per unit of weld length, the type and size of the joint bevel and wire, the shielding gas composition and the CTWD were maintained constant. It was evaluated the surface finish and geometry of the weld beads, the deposition rate and efficiency and the limit welding speeds, resultant from each technique. The results showed that the use of CSC in the trailing wire slightly increases the production capacity of the process, generates less spatter and provides a better visual aspect of the welds, with a narrower $\mathrm{HAZ}$ and $\mathrm{FZ}$, and still keeps the operational maps, which are positive factors. On the other hand, as disadvantages, the use of the CSC led to more convex beads with smaller penetration.
\end{abstract}

Key-words: Tandem GMAW; Current control; Automation.

\section{Introdução}

O processo de soldagem MIG/MAG é um dos mais utilizados na indústria. Demandas por maior produtividade e uma melhor qualidade conduziram para o desenvolvimento de diversas variantes deste processo. O processo MIG Duplo Arame (DA), por exemplo, foi desenvolvido para áreas de aplicação onde altas taxas de deposição são necessárias (comparáveis com as do processo de Arco Submerso), mas em que a flexibilidade do processo MIG/MAG é desejada. De acordo com Lezzi e Costa [1], na soldagem com dois
Este é um artigo publicado em acesso aberto (Open Access) sob a licença Creative Commons Attribution Non-Commerca que permite uso, distribuiçăo e reproduçăo em qual quer meio, sem restriçōes desde que sem fins comerciais e que 0 trabalho original seja corretamente citado. 
arames em arco submerso pode-se atingir taxas de deposição entre 12 a $20 \mathrm{~kg} / \mathrm{h}$, enquanto, para a soldagem com MIG/MAG DA, Nadzam [2] afirma que se pode obter de 9 a $21 \mathrm{~kg} / \mathrm{h}$.

De forma geral, o MIG/MAG DA é a duplicação do processo MIG/MAG com um arame, utilizando duas fontes, dois alimentadores, dois arames e uma única tocha. Esta configuração, em comparação com o processo convencional com um arame, apresenta maior velocidade de soldagem, maior taxa de fusão e maior penetração. De acordo com Hedegård et al. [3], a substituição de MIG/MAG convencional por MIG/MAG DA leva ao aumento da velocidade de soldagem de 50 até 300\%. Já Melton e Muligan [4] afirmam que a taxa de deposição aumenta em até 3 vezes. Este processo pode ser considerado mais econômico, no caso da execução de juntas com numero maior de passes de enchimento (chapas grandes e espessas). Este fato foi observado por Melton e Mulligan [4] no caso da soldagem com MIG/MAG DA da uma chapa de $10 \mathrm{~mm}$ espessura. Os resultados apresentam a redução dos custos totais de $45 \%$, causados pela diminuição dos números de passes (de 5, feitos com MIG/MAG com um arame, para até 2, no caso do uso do MIG/MAG DA).

Do ponto de vista da energização dos arames-eletrodos, segundo Scotti et al. [5], a versão mais utilizada na prática é a como potencial isolado (neste caso, os parâmetros de soldagem são ajustados independentes para cada arame, utilizando-se duas fontes). Os eletrodos podem ser posicionados em paralelo (configuração chamada de Twin) e sequencialmente (um atrás do outro), configuração denominada por Tandem. De forma usual, utiliza-se de corrente contínua constante ou pulsada em ambos os arames. Em ambos os casos, se procura trabalhar com arcos o mais curto possível, para evitar o efeito do sopro magnético e garantir maior estabilidade da poça (menos oscilação da poça, seja devido à uma menor pressão dinâmica do arco, menores arcos menores velocidades do jato, seja devido ao menor momentum das gotas metálicas em transferência, como se pode deduzir por analogia a partir de outras condições apresentadas por Scotti e Rodrigues [6]). Mas um arco muito curto, chegando ao curto-circuito, provocará muito respingos, o que é indesejado.

No caso do uso da corrente pulsada em ambos os arames, pode ser usada a sincronização entre as fontes. Neste caso, a fonte principal (mestre) controla a fonte secundária (escrava) por intermédio de um tempo chamado de defasagem. A sincronização entre as fontes em função das condições de soldagem de cada trabalho é considerada por alguns autores como um fator importante e por outros não. Motta e Dutra [7] afirmam que a defasagem é empregada com o objetivo de reduzir as interações eletromagnéticas entre os arcos, enquanto Goecke et al. [8] declaram que com o uso da corrente pulsada em ambos os eletrodos a sincronização é indispensável. Já no caso da soldagem com correntes altas (corrente média acima do valor da corrente de transição), os resultados de Scotti et al. [5] apresentam que o valor da defasagem não oferece nenhuma redução da atração das gotas e que a recomendação do uso da defasagem na soldagem com corrente pulsada neste caso não e justificada. Esses autores afirmam que soldas de qualidade poderiam ser obtidas com máquinas de soldagem mais convencionais.

A busca do aumento da produtividade e do desempenho do MIG/MAG DA levou à combinação do processo com o laser. Resultados de Reis et al. [9] mostram que esse processo híbrido aumenta a penetração, a largura e a velocidade de soldagem em comparação com o MIG/MAG DA tradicional, que usa em ambos os eletrodos a corrente pulsada. Outra solução para o aumento da produtividade apresentada por Yapp e Blackman [10] na soldagem dos passes de enchimento de dutos foi a possibilidade do uso do MIG/MAG DA em configuração Duplo Tandem GMAW, duplicando assim a taxa de deposição, reduzindo os números de passos e mantendo aproximadamente o mesmo ciclo térmico do MIG/MAG DA.

Considerando-se a constante necessidade de inovação, mesmo para esta versão relativamente recente do MIG/MAG, a empresa Fronius recentemente apresentou uma combinação de arco pulsado no arame-líder com o processo CMT no arame seguidor. Staufer [11] descreve esta abordagem, que foi denominado pelo fabricante de CMT Twin. O CMT é uma das formas de se operar o processo MIG/MAG com curto-circuito controlado, em que a corrente de soldagem é ciclicamente reduzida para valores baixos em períodos de tempo pré-determinados, de tal forma a forçar a gota em formação na ponta do arame a tocar a poça e permitir uma transferência da mesma para a poça por tensão superficial. Ao fim de um tempo para se concluir a transferência, o arame-eletrodo tem sua direção revertida, forçando o rompimento do menisco entre a ponta do arame e a poça. Neste momento, o valor da corrente é restabelecido ao valor original, permitindo a reabertura do arco. Esta forma operacional garante uma transferência bem estável, com um nível baixíssimo de respingos e grande homogeneidade térmica. Este fabricante reivindica que as vantagens do modo operacional com corrente pulsada (menos respingos, melhor penetração) são no processo Twin aliadas com as do CMT, aumentando a produtividade e a profundidade de 
penetração. Rutzinger [12] indica haver também diminuição da interferência magnética entre os eletrodos e do valor de calor aportado. As melhores propriedades de ambos os modos operacionais atuam em um processo novo.

Existem outras formas do processo MIG/MAG em curto-circuito com controle da transferência metálica além do método empregado no CMT. Basicamente, o que se faz é baixar a corrente no momento em que se percebe um curto-circuito (redução da tensão de arco), garantindo-se que a gota seja sugada suavemente pela poça por efeito da tensão superficial. Durante a sugação da gota, não há o aumento progressivo da corrente e das forças a ela conexas, como em curto-circuito convencional. E de forma diferenciada do CMT, o destacamento final da gota é feito pela elevação brusca da corrente ao final do processo de transferência por tensão superficial, a qual neste momento impõe o efeito pinch para concluir o destacamento. Pouco antes de romper a ponte metálica da gota, a corrente é novamente baixada para se evitar um surto de energia ao final de destacamento e, em seguida, aumentada para restabelecer o arco. Esta abordagem que proporciona transferências de alta regularidade e com baixo nível de respingos é aplicada comercialmente pela Lincoln $\left(\mathrm{STT}^{\circledR}\right)$, Miller $\left(\mathrm{RMD}^{\circledR}\right)$, EWM (ColdArc $\left.{ }^{\circledR}\right)$, Kemppi (ArcForce), IMC (CCC), entre outras.

Considerando-se as características do modo operacional curto-circuito-controlado, é de se visualizar que este modo seria muito adequado para soldagem MIG/MAG com DA, por permitir transferência por curto-circuito, consequentemente arco curto (baixa pressão do jato de plasma), mas sem respingos. Por outro lado, a transferência por curto-circuito controlado não é adequada para valores altos da corrente, o que contrasta com o MIG/MAG $\mathrm{DA}$, o qual tem sido aplicado com correntes altas, mais do que $300 \mathrm{~A}$ em cada um dos arames. Mas existem ainda algumas aplicações para processo de soldagem MIG/MAG DA que não foram ainda exploradas em toda sua plenitude, tais como nos passes de enchimento em soldagem orbital. Neste caso, correntes altas não seria um requisito. Desta forma, o objetivo deste trabalho foi avaliar o uso do modo CCC (Curto-circuito controlado) no arame seguidor quanto ao desempenho técnico e econômico do processo MIG/MAG DA.

\section{Procedimento Experimental}

\subsection{Equipamentos e insumos}

Para o desenvolvimento de trabalho, foi utilizada uma tocha de soldagem duplo arame comercial, com os arames paralelos, formando um ângulo de ataque de $90^{\circ} \mathrm{com}$ a superfície da peça de soldagem. Entre os eletrodos foi ajustada uma distância de $15 \mathrm{~mm}$. Para fixação e movimento da tocha, usou-se uma mesa de coordenadas ortogonais $X-Y$, que permite programar trajetórias lineares (ou não) através de sua unidade de controle.

As placas de testes foram confeccionadas de aço carbono ABNT 1020, com as dimensões de $200 \times 52 \times 9,5 \mathrm{~mm}$. Para simular o passe de enchimento, as placas de testes foram chanfradas por fresagem, perfazendo-se um rasgo em formato meia-cana com $4 \mathrm{~mm}$ de profundidade e $10 \mathrm{~mm}$ de largura (Figura 1). Como material de adição, utilizou-se arames-eletrodo da classe AWS ER70S-6, com diâmetro de 1,2 mm. Como gás de proteção, foi usada uma mistura gasosa com composição química nominal de $92 \% \mathrm{Ar}+8 \% \mathrm{CO}_{2^{\prime}}$ a uma vazão de $30 \mathrm{l} / \mathrm{min}$. A distância bico de contato a peça (DBCP) foi mensurada a partir do centro do chanfro e fixada no valor de $21 \mathrm{~mm}$.

Durante todos os experimentos com o MIG/MAG DA no modo Pulsado-Pulsado (DA P-P), foram utilizadas duas fontes de soldagem DIGITEC 600 e dois alimentadores de arame do modelo STA-20. As fontes foram sincronizadas, regulando-se uma defasagem de 5,8 ms entre os pulsos de cada arame. $O$ valor da defasagem foi calculado para que o pulso do arame líder permanecesse durante o tempo de base do arame seguidor. No caso da soldagem com Curto-Circuito Controlado no arame seguidor (MIG/MAG DA P-CCC), utilizou-se a fonte MTE Digitec 600 para arame líder e a fonte DIGIPlus A7 para arame seguidor. A aquisição de sinais de corrente e tensão em cada arame
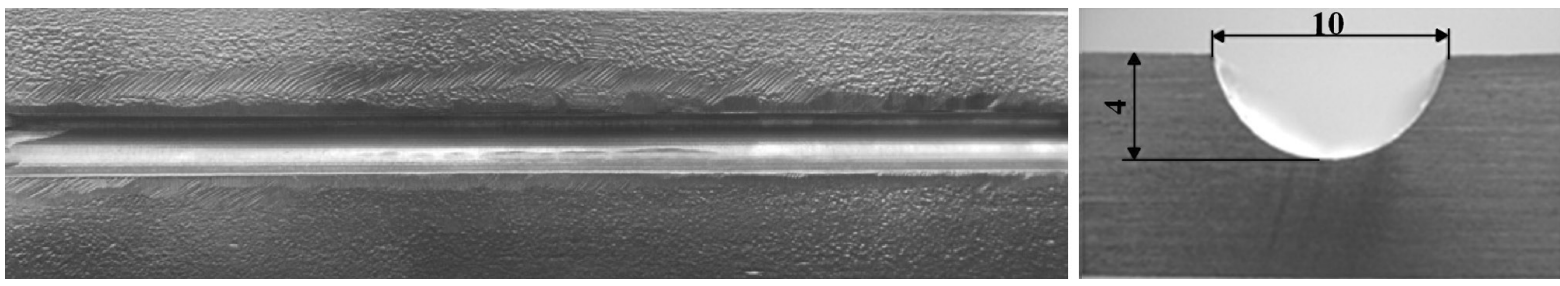

Figura 1. llustração do rasgo confeccionados nas placas de teste para simular uma soldagem de passe de enchimento. 
foi feita através de uma placa AD conectada em um computador via USB. Para visualização dos sinais adquiridos utilizou-se um programa computacional específico Lab VIEW ${ }^{\circledR}$.

Após as soldagens, as placas de teste foram cortadas transversalmente em duas seções e preparadas metalograficamente para revelar a dimensões do cordão (ZF) e da zona afetada pelo calor (ZAC). Após o ataque, as seções foram fotografadas com auxílio de um microscópio digital (usando-se um aumento de $3 \mathrm{x}$ ) e analisadas por um programa computacional de tratamento de imagem (ImageJ). Antes de se efetuar as medições, foi feita a calibração do programa, utilizando-se como base a espessura da chapa. As características geométricas medidas foram a área transversal do cordão, a área da zona afetada pelo calor, a largura do cordão de solda, o reforço e a penetração. Para cada seção foram feitas três medições.

\subsection{Metodologia}

Para fazer a comparação entre as duas abordagens, utilizou-se a mesma corrente média em cada arame

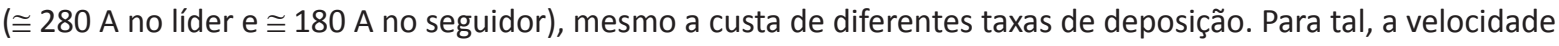
de alimentação foi ajustada em cada arame até se obter as correntes médias desejadas. Outro fator de não variação foi o volume de cordão, mesmo que para isto a velocidades de soldagem, consequentemente, energias de soldagem, tornem-se diferentes. A justificativa para tal abordagem é que numa soldagem típica de duplo arame o que se procura é produção, que pode ser traduzida em volume de material depositado (taxas de deposição) por unidade de comprimento de solda. Tem-se em mente que para os passes de enchimento em soldagens a arco almejam-se duas características:

- Volume de metal adequado (não muito grande, para se evitar altas tensões térmicas e falta de penetração; nem muito pequeno, para não demandar um grande número de passes e seus respectivos tempos mortos);

- Mínima (mas suficiente) penetração no fundo e fusão nas laterais da junta (para minimizar o calor aportado).

Também do ponto de vista comparativo, para se efetuar esta avaliação foram mantidos constantes os seguintes parâmetros: diâmetro e tipo do consumível; gás de proteção; distância bico de contato peça (DBCP); distância entre arames; material e geometria da placa de teste; e chanfro. Como critérios de comparação, foram usados o aspecto superficial do cordão, a geometria do cordão de solda (em relação à penetração, fusão lateral, convexidade do cordão, área total do cordão, área fundida do metal de base e ZAC), aspecto econômico (em relação à geração de respingo e velocidade de soldagem para se fazer a mesma junta) e velocidade limite de soldagem alcançável.

\section{Resultados e Discussões}

\subsection{Soldagem do passe de enchimento com MIG/MAG DA Pulsado-Pulsado (MIG/MAG DA P-P)}

Inicialmente, procurou-se a velocidade de soldagem adequada para se encher o "chanfro" em um único passe, obtendo-se um cordão de solda uniforme e sem falta de enchimento. Esse cordão foi uma velocidade de $7 \mathrm{~mm} / \mathrm{s}$. Para esta velocidade, usou-se uma velocidade de alimentação de 9,7 m/min no arame líder e de 4,6 m/min para o arame seguidor. Em seguida, aumentou-se a velocidade de soldagem até se encontrar a velocidade de soldagem limite máxima do processo na versão MIG/MAG DA P-P. Naturalmente com o aumento da velocidade limite o volume do cordão se tornava menor e não havia mais o requerimento de preenchimento total do chanfro. Considerou-se para definir a velocidade de soldagem limite máxima a condição na qual o acabamento superficial do cordão de solda deixava de ser regular. Aumentou-se a velocidade de soldagem para 26,4 mm/s, $44 \mathrm{~mm} / \mathrm{s}$ e 48,6 mm/s. Os cordões de soldagem ficaram regulares e o processo apresentou um bom comportamento. Aumentou-se, assim, a velocidade de soldagem até $53,5 \mathrm{~mm} / \mathrm{s}$, condição em qual a geometria do cordão de solda começou a ficar irregular. A velocidade anterior foi considerada como velocidade de soldagem limite máxima para este processo.

Os parâmetros de soldagem relativos aos experimentos para a soldagem no modo P-P nas 5 diferentes velocidades de soldagem acima citadas são apresentados na Tabela 1, enquanto a Tabela 2 apresenta os valores de corrente e tensão monitorados durante as soldagens. Oscilogramas de corrente e tensão típicos dessa condição de soldagem está mostrada na Figura 2, demonstrando a característica do processo MIG/MAG DA P-P. Chama-se atenção para o fato de que em soldagem MIG/MAG DA a corrente pulsada não tem a função de controlar a transferência da gota, como no caso do processo MIG/MAG Pulsado convencional. Por isto, os valores de corrente média, e até mesmo os valores de corrente de base, aplicadas no arame 1 estão acima da corrente de transição. 
Tabela 1. Parâmetros regulados para soldagem de passes de enchimento com o MIG/MAG DA P-P.

\begin{tabular}{|c|c|c|c|c|c|c|c|c|c|c|}
\hline \multirow[b]{2}{*}{$\underset{(\mathrm{mm} / \mathrm{s})}{V_{\mathrm{s}}}$} & \multicolumn{5}{|c|}{ Arame 1} & \multicolumn{5}{|c|}{ Arame 2} \\
\hline & $\begin{array}{l}I_{\mathrm{p} 1} \\
(\mathrm{~A})\end{array}$ & $\begin{array}{c}t_{b 1} \\
(\mathbf{m s})\end{array}$ & $\begin{array}{l}I_{b 1} \\
(A)\end{array}$ & $\begin{array}{c}t_{b 1} \\
(\mathrm{~ms})\end{array}$ & $\underset{(m / \mathrm{min})}{\mathrm{V}_{\mathrm{a} 1}}$ & $\begin{array}{l}I_{p 2} \\
(\mathbf{A})\end{array}$ & $\begin{array}{c}t_{p 2} \\
(\mathrm{~ms})\end{array}$ & $\begin{array}{l}I_{b 2} \\
(A)\end{array}$ & $\begin{array}{c}t_{b 2} \\
(\mathrm{~ms})\end{array}$ & $\begin{array}{c}V_{\mathrm{a} 2} \\
(\mathrm{~m} / \mathrm{min})\end{array}$ \\
\hline $\begin{array}{c}7 ; 26,4 ; 44 ; \\
48,6 ; 53,5\end{array}$ & 366 & 3,5 & 255 & 8 & 10,3 & 360 & 3,5 & 106 & 8 & 4,4 \\
\hline
\end{tabular}

Nota: Vs - velocidade de soldagem; Ip1 - Corrente de pulso arame 1; tp1 - tempo de pulso arame 1, lb1 - corrente de base arame 1; tb1 - tempo de base arame1; Va1 - velocidade de alimentação arame 1; Ip2 - Corrente de pulso arame 2; tp2 - tempo de pulso arame 2, lb2 - corrente de base arame 2; tb2 - tempo de base arame2; Va2 - velocidade de alimentação arame 2.

Tabela 2. Parâmetros monitorados durante soldagem de passe de enchimento com o MIG/MAG DA P-P.

\begin{tabular}{|c|c|c|c|c|c|c|c|}
\hline $\begin{array}{l}V_{\text {s regulada }} \\
(\mathrm{mm} / \mathrm{s})\end{array}$ & $\mathrm{V}_{\mathrm{s} \text { real }}(\mathrm{mm} / \mathrm{s})$ & Arame & $\begin{array}{c}V_{\text {a real }} \\
(\mathrm{m} / \mathrm{min})\end{array}$ & $\underset{(A)}{I_{M}}$ & $I_{\text {RMS }}(\mathrm{A})$ & $U_{M}(\mathbf{V})$ & $\mathbf{U}_{\mathrm{RMS}}(\mathbf{V})$ \\
\hline \multirow[t]{2}{*}{7} & 7,2 & Arame 1 & 9,7 & 282,9 & 287,4 & 27,9 & 28,2 \\
\hline & & Arame 2 & 4,6 & 174,3 & 207,5 & 22,5 & 23,2 \\
\hline \multirow[t]{2}{*}{26,4} & 25,5 & Arame 1 & 9,7 & 282,3 & 285,9 & 26,8 & 27 \\
\hline & & Arame 2 & 4,6 & 173,3 & 207,6 & 20 & 22,9 \\
\hline \multirow[t]{2}{*}{44} & 42 & Arame 1 & 9,7 & 282 & 286,3 & 25,7 & 25,6 \\
\hline & & Arame 2 & 4,6 & 174,2 & 206,1 & 22,8 & 23,6 \\
\hline \multirow[t]{2}{*}{48,6} & 46,4 & Arame1 & 9,7 & 284,9 & 288,9 & 25,5 & 25,9 \\
\hline & & Arame 2 & 4,6 & 179,1 & 213,2 & 21,7 & 22,5 \\
\hline \multirow[t]{2}{*}{53,5} & 51 & Arame 1 & 9,7 & 281,9 & 286,3 & 26,4 & 26,5 \\
\hline & & Arame 2 & 4,6 & 165,8 & 200,3 & 26,5 & 25,2 \\
\hline
\end{tabular}

Nota: Vs regulada - velocidade de soldagem regulada; Vs real - velocidade de soldagem real; Va real - velocidade de alimentação real Im - corrente media; $I_{\text {RMS }}$ - corrente RMS; Um - tensão media; $U_{\text {RMS }}$ - tensão RMS.

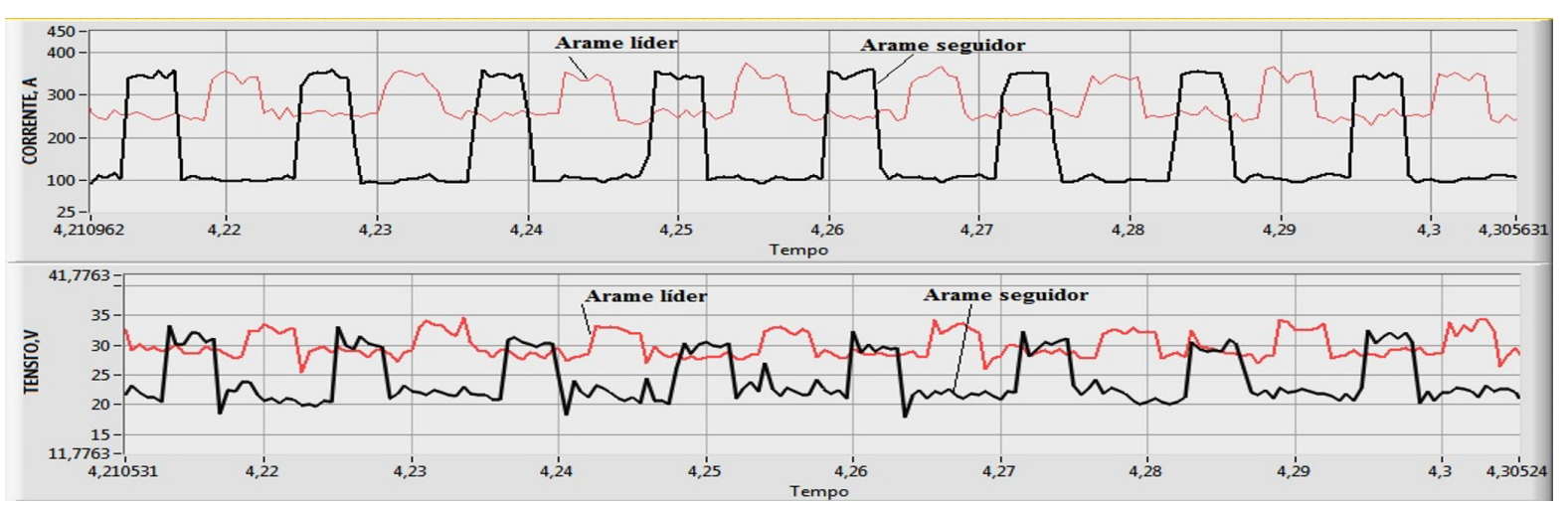

Figura 2. Oscilograma típica de corrente e tensão para a soldagem com os parâmetros da Tabela 1 (MIG/MAG DA P-P).

A Figura 3, por seu lado, ilustra os cordões resultantes de todos estes experimentos. Como se pode observar, o cordão de solda executado com velocidade de soldagem de $7 \mathrm{~mm} / \mathrm{s}$ tem uma boa aparência e isenta de descontinuidades. Os demais cordões apresentam piora crescente do acabamento superficial (rugosidade macro e desvios de paralelismo das bordas), mas ainda garantindo a penetração mínima necessária e não presença de descontinuidades (como mordedura, descontinuidade do cordão). As seções transversais dos cordões de soldas apresentam uma penetração boa e suficiente, tanto no fundo quanto na lateral do cordão. Pode-se observar que o cordão de solda não apresenta falta de fusão em nenhum dos casos, seja a velocidade de soldagem baixa ou alta. A macrografia dos cordões de soldas foi feita só para as soldas que não apresentaram descontinuidades do cordão e quais foram consideradas aceitáveis. 


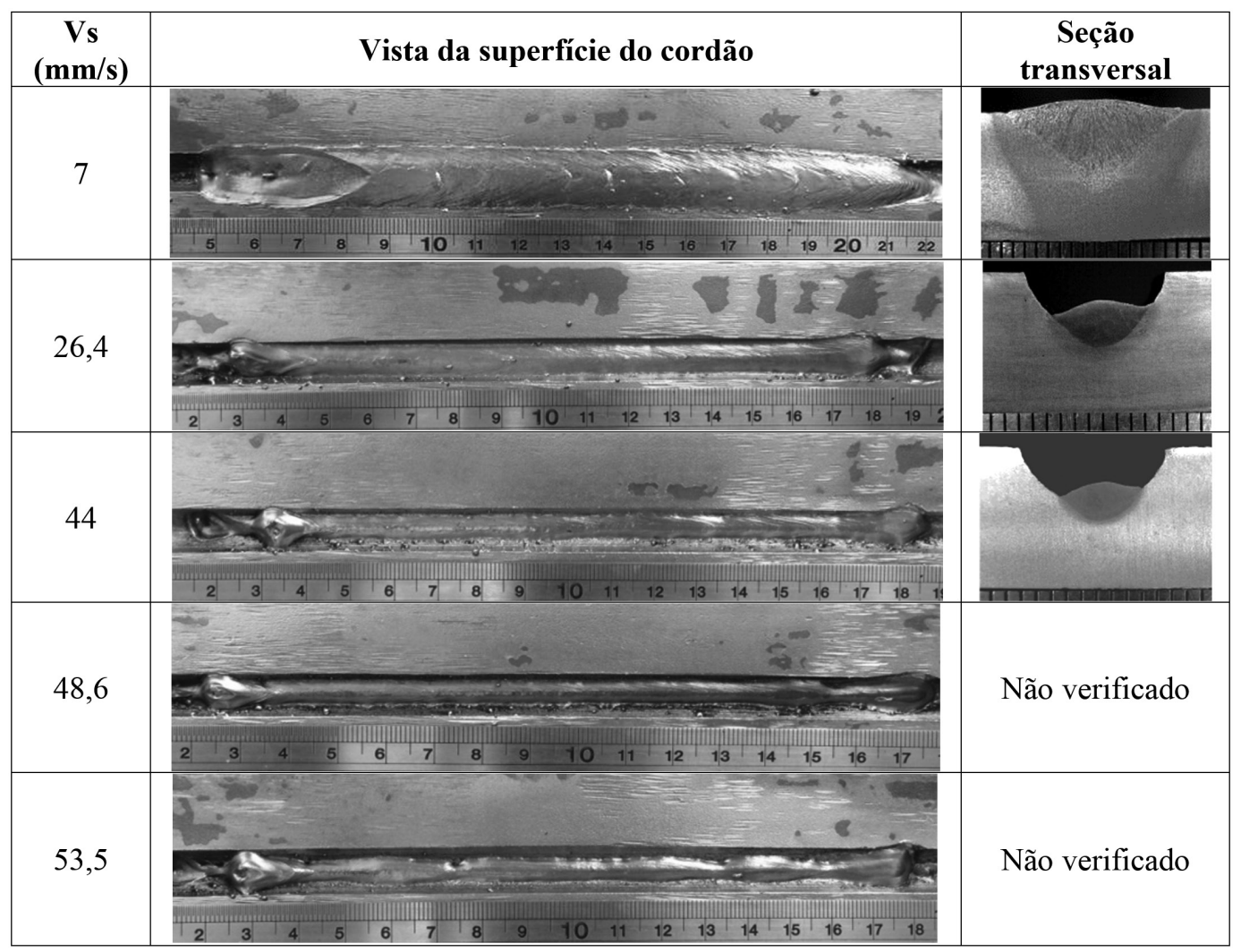

Figura 3. Aspecto visual dos cordões das soldagens do passe de enchimento e as respectivas seções transversais com processo MIG/MAG DA P-P.

\subsection{Soldagem passe de enchimento com MIG/MAG DA Pulsado-Curto-Circuito Controlado (MIG/MAG DA P-CCC)}

No caso da combinação dos modos operacionais MIG/MAG DA P-CCC, os parâmetros utilizados para o arame líder foram os mesmos dos da soldagem com MIG/MAG DA P-P. Para o arame seguidor, no qual se utilizou o curto-circuito controlado, precisavam-se encontrar os parâmetros adequados para soldagem. Porém, de forma mais intensa do que no modo pulsado, o número total dos parâmetros que precisam ser regulados chega a 16. A onda do modo operacional por curto-circuito controlado esta apresentada na Figura 4.

Reproduziu-se as soldas feitas com MIG/MAG DA P-P, mantendo-se o mesmo volume de material depositado e os valores das correntes médias. Mas para se atingir a mesma corrente média no arame seguidor, a velocidade de alimentação do arame foi um pouco maior com CCC do que com Pulsado (5,0 contra 4,6 m/min). Este resultado mostra que o controle da corrente em CCC proporciona maior taxa de fusão do que em pulsado para mesma corrente média. Outra hipótese é de que durante a soldagem com MIG/MAG DA P-CCC não foi utilizada a sincronização, fato que pode conduzir a uma melhor transferência energética do arame líder para o arame seguidor. Consequentemente, para se manter o mesmo volume de material depositado por unidade de comprimento de solda, as velocidades de soldagem sofreram um pequeno aumento em relação ao modo MIG/MAG DA P-P. As Tabelas 3 e 4 apresentam os parâmetros de regulagem e monitorados aplicados nos experimentos, enquanto a Figura 5 apresenta oscilogramas de corrente e tensão típicos dessa condição de soldagem, demonstrando a característica do processo MIG/MAG DA P-CCC. Observou-se visualmente que o arco do arame-seguidor, onde foi utilizado o CCC, é menor do que no caso da soldagem com MIG/MAG DA P-P, sugerindo uma estabilidade superior do processo.

A caracterização visual dos cordões de soldas é apresentada na Figura 6. As seções transversais dos cordões de solda não apresentam falta de fusão e oferecem uma penetração adequada. Quando se soldou com a velocidade máxima limite do processo DA P-P (53,5 mm/s), calculada para o mesmo volume para o processo MIG/MAG DA 


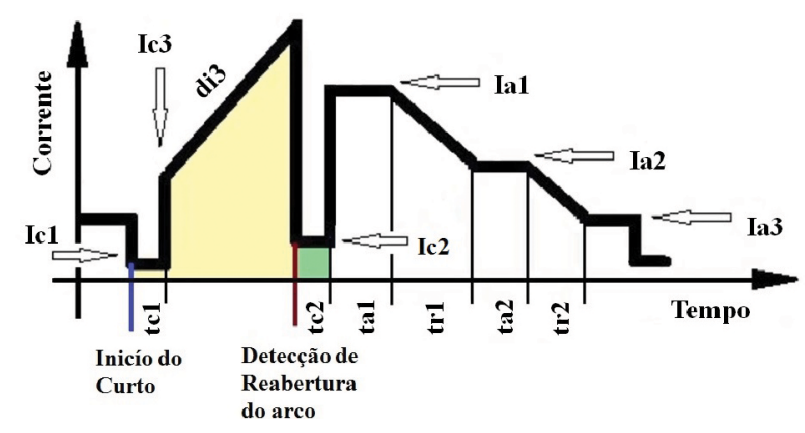

Figura 4. Onda do modo operacional CCC.

Tabela 3. Parâmetros regulados para soldagem de passe de enchimento com MIG/MAG DA P-CCC.

\begin{tabular}{|c|c|c|c|c|c|c|c|}
\hline \multicolumn{8}{|c|}{ Arame 1} \\
\hline \multicolumn{2}{|c|}{$V_{s}(\mathrm{~mm} / \mathrm{s})$} & $I_{p 1}(A)$ & $t_{b 1}(m s)$ & $I_{b 1}(A)$ & $t_{b 1}(m s)$ & \multicolumn{2}{|c|}{$\mathrm{V}_{\mathrm{a} 1}(\mathrm{~m} / \mathrm{min})$} \\
\hline \multicolumn{2}{|c|}{7,$2 ; 27,2 ; 45,3 ; 50 ; 55$} & 350 & 3,5 & 250 & 8,0 & & \\
\hline \multicolumn{8}{|c|}{ Arame 2} \\
\hline la1 (A) & $\operatorname{ta} 1$ (ms) & la2 (A) & ta2 (ms) & Va (m/min) & Ic1 (A) & tc1 (ms) & Ic2 (A) \\
\hline 290 & 2,0 & 190 & 3,0 & 5,3 & 95 & 0,5 & 105 \\
\hline tc2 (ms) & Ic3 (A) & di3 (A/s) & $\operatorname{Ucc}(\mathrm{V})$ & kr & $\operatorname{tr} 1$ (ms) & $\operatorname{tr} 2$ (ms) & $\operatorname{la3}(\mathrm{A})$ \\
\hline 0,6 & 214 & 120 & 10 & 1,8 & 0,6 & 0,6 & 140 \\
\hline
\end{tabular}

Nota: Vs - velocidade de soldagem la 2 - corrente de pulso de arco; la 2 - corrente para aumento da taxa de fusão; ta 2 - tempo da corrente $\mathrm{la}_{1} 2 ; \mathrm{ta} 2$ - tempo da corrente $\mathrm{Ia}_{2} 2 ; \mathrm{la}_{3} 2$ - corrente de manutenção do arco; $\mathrm{di}_{3} 2$ - taxa de variação de subida da corrente durante o curto em A/ms; $\operatorname{tr}_{1} 2$ - tempo de rampa do patamar 1 para o patamar $2 ; \operatorname{tr}_{2} 2$ - tempo de rampa do patamar 2 para o patamar $3 ; \operatorname{tc}_{1} 2$ - tempo de assentamento da gota; $\mathrm{tc}_{2} 2$ - tempo de espera de reabertura; $\mathrm{Ic}_{1} 2$ - corrente de assentamento de gota; $\mathrm{Ic}_{2} 2$ - corrente de rompimento da ponte metálica.

Tabela 4. Parâmetros monitorados para soldagem de passe de enchimento com MIG/MAG DA P-CCC.

\begin{tabular}{|c|c|c|c|c|c|c|c|}
\hline $\begin{array}{l}V_{\text {s regulada }} \\
(\mathrm{mm} / \mathrm{s})\end{array}$ & $\begin{array}{c}V_{\text {s real }} \\
(\mathrm{mm} / \mathrm{s})\end{array}$ & Arame & $\begin{array}{c}V_{\text {a real }} \\
(\mathrm{m} / \mathrm{min})\end{array}$ & $I_{m}(A)$ & $I_{r m s}(A)$ & $U_{m}(V)$ & $\mathbf{U}_{\mathrm{rms}}(\mathrm{V})$ \\
\hline \multirow[t]{2}{*}{7,2} & 7,4 & Arame 1 & 9,7 & 283,4 & 288,1 & 26,7 & 27 \\
\hline & & Arame 2 & 5,0 & 173,4 & 185,2 & 19,4 & 21,2 \\
\hline \multirow[t]{2}{*}{27,2} & 26,2 & Arame 1 & 9,7 & 281,4 & 285,9 & 29,3 & 29,5 \\
\hline & & Arame 2 & 5,0 & 176,2 & 187,2 & 19,1 & 20,3 \\
\hline \multirow[t]{2}{*}{45,3} & 43,3 & Arame 1 & 9,7 & 282,7 & 287,2 & 26,4 & 26,6 \\
\hline & & Arame 2 & 5,0 & 175,9 & 186,9 & 18,9 & 20,2 \\
\hline \multirow[t]{2}{*}{50} & 47,7 & Arame 1 & 9,7 & 282,2 & 286,7 & 27,5 & 27,7 \\
\hline & & Arame 2 & 5,0 & 169,5 & 180,8 & 18,4 & 19,8 \\
\hline \multirow[t]{2}{*}{55} & 61,9 & Arame 1 & 9,7 & 284,6 & 289,8 & 27,1 & 27,6 \\
\hline & & Arame 2 & 5,0 & 166,1 & 181,1 & 20,8 & 24,1 \\
\hline
\end{tabular}

Nota: Vs regulada - velocidade de soldagem regulada; Vs real - velocidade de soldagem real; Va real - velocidade de alimentação real; Im - corrente media; $I_{R M S}$ - corrente RMS; Um - tensão media; $U_{\text {RMS }}$ - tensão RMS.

P-CCC como $55 \mathrm{~mm} / \mathrm{s}$, o comportamento do processo e o formato do cordão também apresentou resultados inaceitáveis. Diminuindo-se a velocidade de soldagem até $50 \mathrm{~mm} / \mathrm{s}$ obteve-se um cordão de solda regular e considerou-se essa velocidade como a velocidade máxima limite.

\subsection{Discussão e comparação dos resultados na soldagem de um passe de enchimento}

\subsubsection{Aspecto visual dos cordões de solda}

Analisando-se os cordões de soldas, pode-se afirmar que todas as soldas feitas com ambas versões do processo são aceitáveis menos as soldas feitas com a velocidade de soldagem $53,5 \mathrm{~mm} / \mathrm{s}$ pelo processo MIG/MAG DA P-P e 


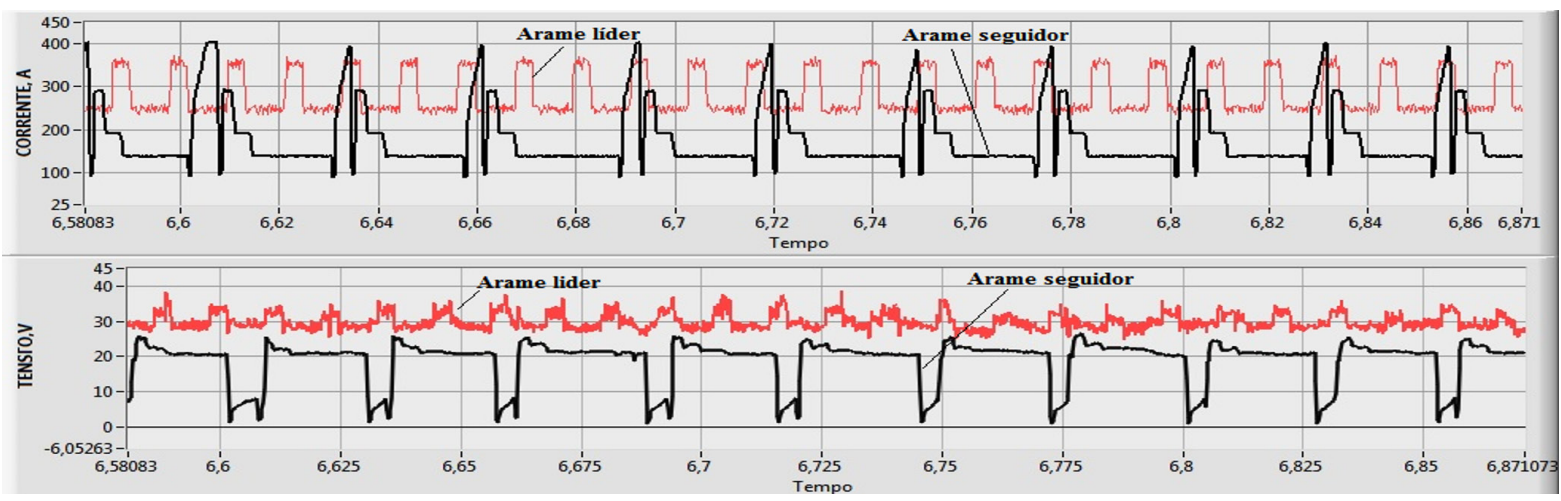

Figura 5. Oscilograma típica de corrente e tensão para a soldagem com os parâmetros da Tabela 4 (MIG/MAG DA P-CCC).

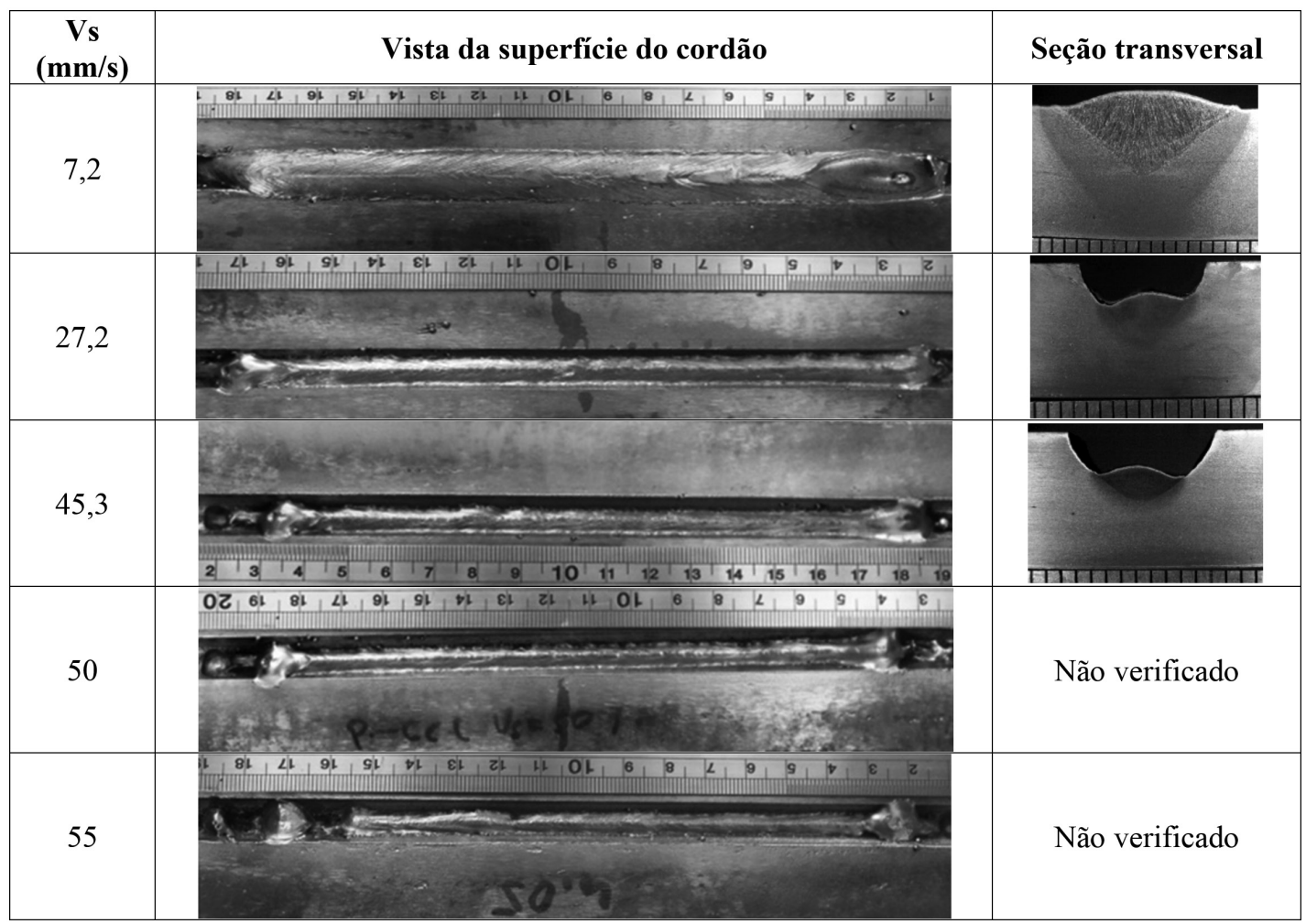

Figura 6. Aspecto visual dos cordões de soldas das soldagens do passe de enchimento e as respectivas seções transversais com processo MIG/MAG DA P-CCC.

$55 \mathrm{~mm} / \mathrm{s}$ no caso do MIG/MAG DA P-CCC. O modo DA P-P apresenta uma quantidade um pouco maior de respingos. No caso do MIG/MAG DA P-CCC, o cordão de solda ficou um pouco mais convexo, o que ocorre devido ao tamanho do arco; no caso do CCC, o arco é visualmente menor em comparação com o P, confirmado pelos maiores valores de tensão do P. O cordão de solda feito com MIG/MAG DA P-P em velocidade baixa apresenta algumas ondas na superfície do cordão, enquanto no caso do MIG/MAG DA P-CCC o cordão fica mais liso.

\subsubsection{Faixa operacional da velocidade de soldagem (Vs)}

Figura 7 apresenta as faixas operacionais das velocidades de soldagem resultantes do uso dos dois modos operacionais para as condições (parametrização) estipuladas. Também nesta figura é apresentada a faixa de material depositado para cada versão do processo dentro de cada envelope operacional. Pode-se observar que 
ambos os modos operacionais apresentam aproximadamente o mesmo envelope de operação e a mesma faixa de material depositado por unidade de comprimento do cordão.

\subsubsection{Geometria dos cordões de soldas}

A avaliação da geometria dos cordões de soldas obtidos com as 2 versões do processo de soldagem foi feita para os cordões de soldas com o mesmo volume depositado. No caso, a solda feita com MIG/MAG DA P-P a uma velocidade de soldagem de $7 \mathrm{~mm} / \mathrm{s}$ foi comparada com a solda com MIG/MAG DA P-CCC na velocidade de soldagem $7,2 \mathrm{~mm} / \mathrm{s}$, ou a com $26,4 \mathrm{~mm} / \mathrm{s}$ com a de $27,2 \mathrm{~mm} / \mathrm{s}$ e a com $44 \mathrm{~mm} / \mathrm{s}$ com a de $45,3 \mathrm{~mm} / \mathrm{s}$. Para facilitar a comparação, as seções dos cordões de soldas para essas velocidades estão apresentadas reapresentadas na Figura 8. Como se pode perceber, as velocidades de soldagem para MIG/MAG DA P-CCC é ligeiramente maior, em média $3 \%$ maior.

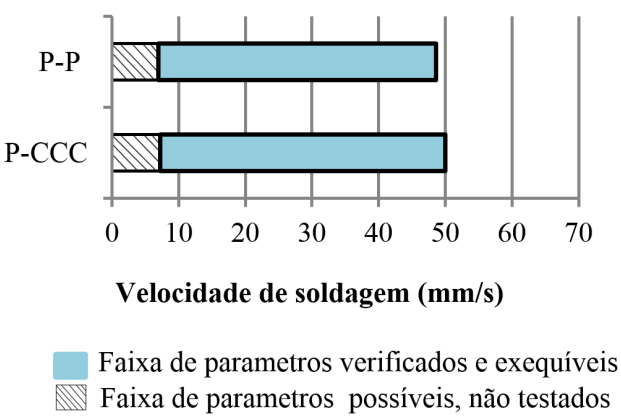

(a)

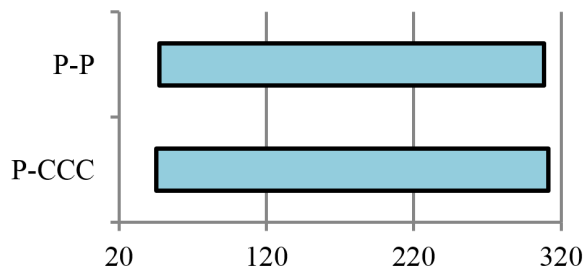

Quantidade de material depositado (g/m)

(b)

Figura 7. Representação da faixa operacional: a) velocidades de soldagem limites; b) quantidade de material depositado por unidade de comprimento de cordão.

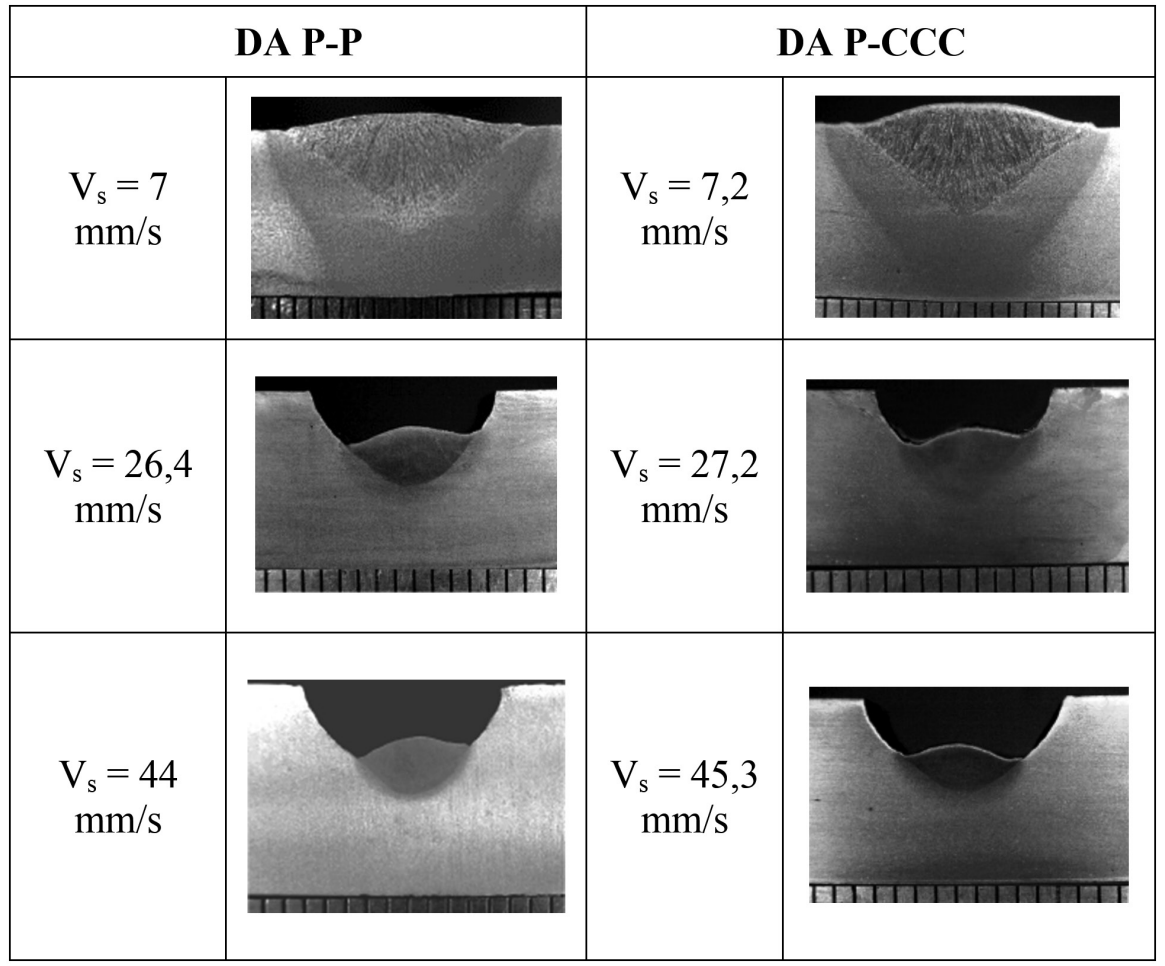

Figura 8. Seções transversais dos cordões de soldas para uma mesma quantidade de material depositado por unidade de comprimento de cordão e mesma corrente média em cada arame. 


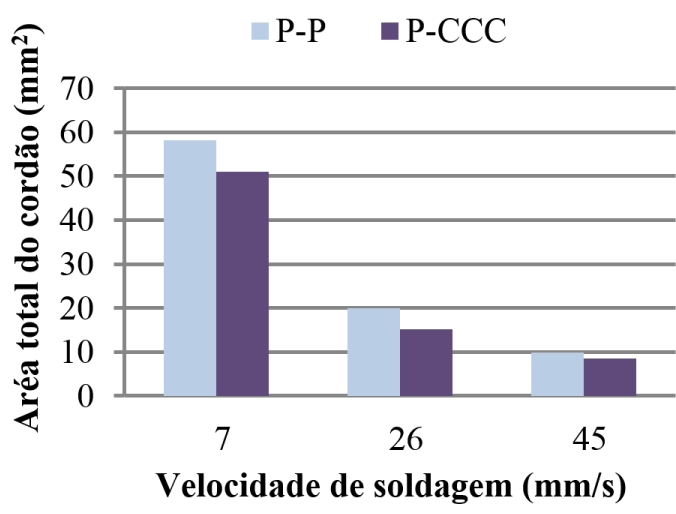

Figura 9. Comparação entre as áreas totais dos cordões feitos pelos modos operacionais MIG/MAG DA P-P e MIG/MAG DA P-CCC a uma mesma corrente média e mesma área adicionada por unidade de comprimento do cordão de solda (média de duas seções transversais por modo operacional).

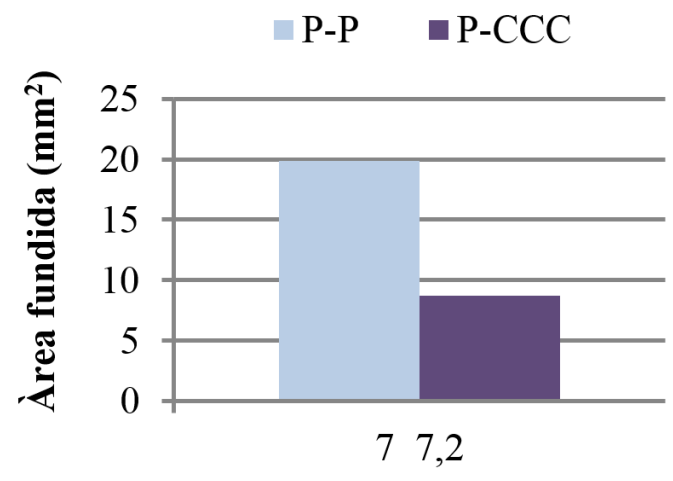

Velocidade de soldagem $(\mathrm{mm} / \mathrm{s})$

Figura 10. Resultados do cálculo da área fundida do metal de base (média de duas seções transversais por modo operacional).

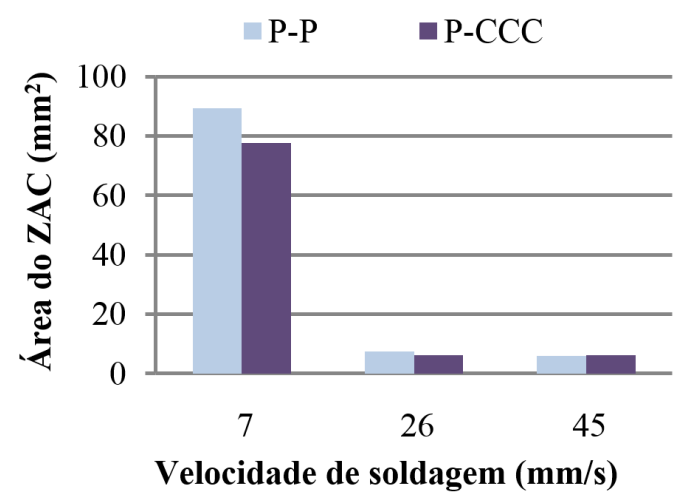

Figura 11. Áreas das ZACs dos cordões de solda (média de duas seções transversais por modo operacional).
Na Figura 9 são apresentadas as áreas totais dos cordões de solda para as condições acima definidas na Figura 8, ou seja, para uma mesma corrente média e mesmo volume de material depositado por unidade de comprimento de solda (mesma área depositada). A característica de se ter uma velocidade de soldagem ligeiramente maior indica uma leve tendência de maior produtividade ao MIG/MAG DA P-CCC, se for levado em conta apenas o tempo de soldagem para se fazer o mesmo cordão (menor tempo de soldagem, menor custo de mão de obra e de consumo de gás, etc.). Além disto, como se pode observar, o modo operacional MIG/MAG DA P-P apresenta uma área total do cordão maior (em valores absolutos, as diferenças se tornam progressivamente menores para maiores velocidades de soldagem).

A maior área total do cordão obtida com MIG/MAG DA P-P se deve ao fato de que, devido à menor velocidade de soldagem e por causa do tipo do arco seguidor, a chapa recebe mais calor, apresentando uma energia de soldagem maior. Uma área do cordão maior pode ser um indício de maiores tensões térmicas.

Efetuado agora o cálculo da área fundida do metal de base (da área total do cordão medida, subtraiu-se a área do chanfro e a área do reforço) para a velocidade de soldagem em qual o chanfro foi completamente preenchido $(7 \mathrm{~mm} / \mathrm{s}$ para MIG/MAG DA P-P e 7,2 mm/s para MIG/MAG DA P-CCC). Pode-se confirmar pela Figura 10, de forma mais clara, que o modo DA P-P apresenta uma área fundida maior.

Já a Figura 11 apresenta as áreas das ZACs dos cordões de solda, também para as condições de uma mesma corrente média e mesmo volume de material depositado por unidade de comprimento de solda (mesma área depositada). Da mesma forma, o modo DA P-P apresenta um ZAC maior, principalmente em baixas velocidades de soldagem (maior energia), quando as restrições ao fluxo de calor pela geometria da chapa se tornam mais críticas.

Mas a área fundida por si não reflete a configuração geométrica do cordão. Por isto, torna-se também importante as medições da penetração, largura e reforço dos cordões de solda, como ilustra Figura 12. As medições mostram que no caso da soldagem com o modo DA P-P obteve-se uma penetração maior. Este fato era esperado, uma vez que a corrente pulsada em comparação com o curto-circuito controlado, além de proporcionar uma maior energia de soldagem, tem o efeito da transferência das gotas (momentum) do arame seguidor. Outro fator que influencia a penetração é o jato de plasma do arame seguidor, que permite abrir e empurrar poça metálica e transferir mais calor para dentro da chapa, resultando em 


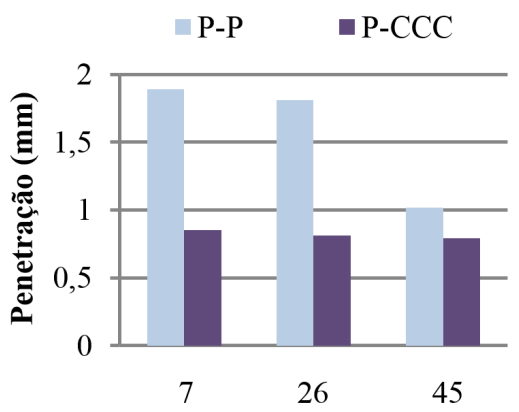

Velocidade de soldagem $(\mathrm{mm} / \mathrm{s})$

(a)

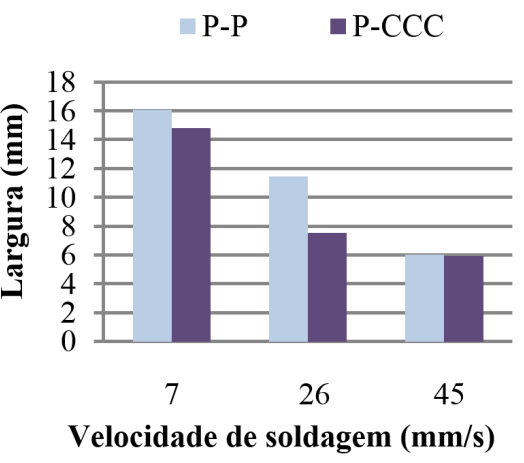

(b)

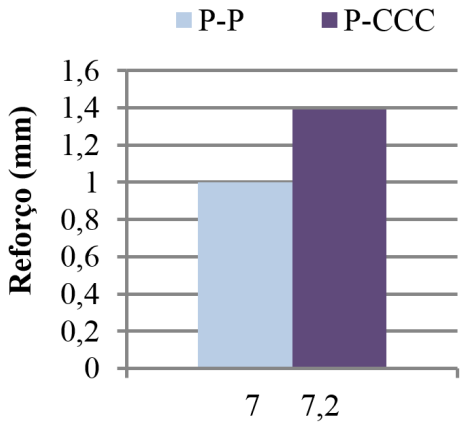

Velocidade de soldagem $(\mathrm{mm} / \mathrm{s})$

(c)

Figura 12. Influência do modo operacional sobre a geometria do cordão: (a) penetração; (b) largura; e (c) reforço (média de duas seções transversais por modo operacional).

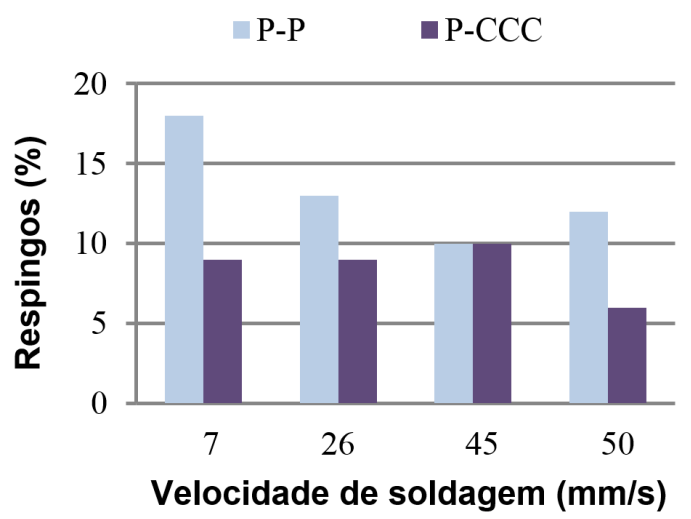

Figura 13. Geração de respingos para diferentes velocidades de soldagem durante os testes comparativos com os processos MIG/MAG DA P-P e MIG/MAG DA P-CCC.

uma penetração maior. Ao se comparar as correntes $I_{\text {rms }}$ nos arames seguidores, pôde-se observar que a corrente $\mathrm{I}_{\mathrm{rms}}$ do processo pulsado é maior em aproximadamente 20 A. Esta diferença também pode influenciar sobre o tamanho da penetração.

Outra forma de avaliar geometricamente o cordão é pela convexidade do mesmo, representada pela relação reforço e largura (quanto maior esta relação, mais convexo o cordão). Pode-se observar pela Figura 13 que o cordão com o MIG/MAG DA P-CCC tem o reforço maior e a largura menor (mais convexo). Essa situação era de esperar, baseando-se no fato de que em MIG/MAG DA seria o arame seguidor que governa o acabamento do cordão e do modo CCC se espera um menor comprimento de arco, além de não ficar $100 \%$ com arco aceso (reduz a molhabilidade).

\subsubsection{Avaliação econômica}

Como já frisado, a diferença das velocidades de soldagem entre os processos não é significativa, o que conduz a um tempo de execução e um consumo do gás de proteção aproximadamente iguais. Além disto, com o MIG/MAG DA P-CCC a taxa de deposição é um pouco maior, mas balanceando-se com a velocidade de soldagem que também é um pouco maior, a taxa de deposição de ambos modos operacionais se tornam iguais. Entretanto, calculada a eficiência de deposição (diferença de volume das chapas antes e após soldagem), expresso pelo percentual de respingos na Figura 13, percebe-se que o modo DA P-CCC tem uma porcentagem de respingos 
menor, em média de 8\%, em comparação com modo MIG/MAG DA P-P, em torno 13\%. Pode-se afirmar, pois, que no caso do MIG/MAG DA P-CCC a operação de limpeza dos respingos seria minimizada.

Outro aspecto que merece destaque, apesar de não tanto significativo, é o valor da tensão do segundo arco. O modo MIG/MAG DA P-CCC tem uma tensão menor (aproximadamente 2-3 V), o que leva a um consumo de energia menor. Desta forma, há evidências de que o modo MIG/MAG DA P-CCC é economicamente melhor do que o modo MIG/MAG DA P-P.

\section{Conclusões}

Tendo em vista o objetivo do trabalho, o de avaliar o uso do Curto-Circuito Controlado (CCC) no arame seguidor na soldagem de passes de enchimento na posição plana com o processo MIG/MAG DA, mantendo-se os mesmos valores das correntes médias (280 A para arame líder e $180 \mathrm{~A}$ para arame seguidor) e o mesmo volume do cordão por unidade de comprimento de solda, pode-se concluir que o uso do CCC em substituição ao uso da corrente pulsada (mais tradicional) é capaz de apresentar vantagens econômicas (uma leve tendência de maior produtividade e uma quantidade menor de respingos gerados), melhor acabamento do cordão, menor área fundida do metal de base e ZACs menores, sem prejuízo para a faixa operacional da velocidade de soldagem, embora com menor penetração do cordão e oferecer soldas mais convexas.

\section{Agradecimentos}

Os autores gostariam de agradecer ao programa de Pós-Graduação da UFU pela oportunidade para realização do trabalho. Também ao CNPq, FAPEMIG e CAPES, pelo fornecimento de bolsa de pesquisa e de bolsas de estudo (mestrado). Adicionalmente, gostariam de agradecer ao Grupo Centro para Pesquisa e Desenvolvimento de Processos de Soldagem - LAPROSOLDA pela disponibilização de equipamentos insumos, e à White Martins pelo fornecimento do gás de proteção.

\section{Referências}

[1] Lezzi F, Costa L. The development of conventional welding process in naval construction. Welding International. 2013;27(10):786797. http://dx.doi.org/10.1080/09507116.2012.753256.

[2] Nadzam J. Tandem GMAW: the flexibility of pulsed spray transfer. Welding Innovation. 2002;19(2).

[3] Hedegård J, Andersson J, Tolf E, Weman K, Lundin M. Enhanced prospects for tandem-MIG/MAG welding. Quebec: IIW; 2004. (International Institute of Welding Document, $n$. XII-1808-04).

[4] Melton GB, Mulligan SJ. Tandem MIG welding for improved productivity. In: Proceedings of the EUROJOIN4 - 4TH European Conference on Welding, joining and cutting. Development of welding and allied processes at the beginning of the new millennium; 2001 Maio 24-26; Cavtat-Dubrovnik, Croatia. Cavtat-Dubrovnik: TWI; 2001. p. 313-318.

[5] Scotti A, Morais CO, Vilarinho LO. The effect of out-of-phase pulsing on metal transfer in twin-wire GMA welding at high current level. Welding Journal. 2006;(October):225s230s.

[6] Scotti A, Rodrigues CEAL. Determination of momentum as a mean of quantifying the mechanical energy delivered by droplets during MIG/MAG welding. The European Physical
Journal Applied Physics. 2009;45(1):11201. http://dx.doi. org/10.1051/epjap:2008196.

[7] Motta MF, Dutra JC. Efeitos das variáveis do processo MIG/ MAG duplo arame com potenciais isolados nas características geométricas do cordão de solda. Soldagem \& Inspeção. 2005;10(1):22-30.

[8] Goecke S, Hedegard J, Lundin M. Tandem MIG/MAG Welding. Svetsaren. 2001;2-3:24-28.

[9] Reis RP, Norrish J, Cuiuri D. Preliminary evaluation on lasertandem GMAW. Welding in the World. 2011;55(9-10):41-49. http://dx.doi.org/10.1007/BF03321319.

[10] Yapp D, Blackman SA. Recent developments in high productivity pipeline welding. Journal of the Brazilian Society of Mechanical Sciences and Engineering. 2004;26(1):89-97. http://dx.doi. org/10.1590/S1678-58782004000100015.

[11] Staufer, H. CMT-Twin-Expanding GMA-Tandem welding. Quebec: IIW; 2012. (International Institute of Welding Document, $\mathrm{n}$. XII-2073-12).

[12] Rutzinger B. Influence of the welding process to the dilution rate of weld overlays on unalloyed steel using the weld consumable ERNiCrMo-3 (Alloy 625). Biuletyn Instytutu Spawalnictwa w Gliwicach. 2014;58(5):72-75. 